\title{
Conclusions du rapporteur public
}

Tribunal administratif de Rouen, M. et $\mathrm{M}^{\text {me }}$ B. et $\mathrm{M}^{\text {lle }} \mathrm{B}$., $\mathrm{n}^{\circ} 1003110$

Rapporteur Public's Conclusions on Case no. 1003110, Rouen Tribunal

Administratif, Mr and Mrs B and Ms B

\section{Gilles Armand}

\section{OpenEdition \\ Journals}

Édition électronique

URL : http://journals.openedition.org/add/779

DOI : $10.4000 /$ add. 779

ISSN : 2606-1988

\section{Éditeur}

Presses universitaires de Rouen et du Havre

\section{Édition imprimée}

Date de publication : 1 avril 2015

Pagination : 27-34

ISSN : 1955-0855

Référence électronique

Gilles Armand, «Conclusions du rapporteur public », Les Annales de droit [En ligne], 7 | 2013, mis en ligne le 03 mai 2018, consulté le 20 avril 2019. URL : http://journals.openedition.org/add/779 ; DOI 10.4000/add. 779

Presses universitaires de Rouen et du Havre 


\title{
Conclusions du rapporteur public
}

\author{
Tribunal administratif de Rouen, \\ $M$. et $M^{\text {me }}$ B. et $M^{\text {lle }}$ B., $n^{\circ} 1003110$
}

Gilles Armand

M. et $\mathrm{M}^{\mathrm{me}} \mathrm{B}$. ont adopté en 1998 deux enfants, S. et E., respectivement nées les 2 décembre 1987 et 13 avril 1989 en Pologne. Sur décision du procureur de la République du 6 février 2004 fondée sur l'article 375-5 du code civil, en raison des fugues la mettant en danger, E., alors âgée de quatorze ans a fait l'objet d'un placement provisoire et été confiée à l'aide sociale à l'enfance du département de l'Eure. Après avoir été hospitalisée le 9 février 2004 à Évreux en raison d'une tentative de suicide, suivie le 10 février d'une seconde menace de mettre fin à ses jours, elle a quitté l'hôpital le 13 février 2004 dans un état léthargique lié au traitement administré les jours précédents. Le soir de ce 13 février, alors qu'elle était placée auprès du foyer départemental de l'enfance d'Évreux, elle a quitté ce lieu en compagnie d'une autre adolescente afin de se rendre dans un hôpital désaffecté où les jeunes filles de son lieu d'hébergement et les garçons du foyer du Bois-Verlande, annexe du foyer départemental de l'enfance, avaient l'habitude de se rencontrer. Après une relation sexuelle consentie avec un mineur présenté comme son petit ami, elle a fait l'objet de relations sexuelles non consenties avec trois autres garçons qui avaient pu quitter leur foyer. Le 16 février 2004, le parquet d'Évreux a été destinataire d'un signalement transmis par le foyer départemental de l'enfance de cette ville, faisant état des viols commis dans la nuit du 13 au 14 février 2004 sur E. B., commis par quatre pensionnaires du foyer du Bois-Verlande. Une instruction pour viol commis sur la personne d'un mineur de quinze ans en réunion, complicité de viol commis sur la personne d'un mineur de quinze ans en réunion, nonassistance à personne en danger et non-dénonciation de crime ayant été ouverte, dans le cadre de laquelle $\mathrm{M}$. et $\mathrm{M}^{\mathrm{me}} \mathrm{B}$. ont déposé plainte 
avec constitution de partie civile, E. B. a été auditionnée dans le cadre de cette instruction le 12 avril 2005 puis s'est suicidée le 17 mai suivant. Le 18 février 2010, la cour d'appel de Rouen a confirmé l'ordonnance de non-lieu rendue le 29 octobre 2009 par le juge d'instruction du tribunal de grande instance d'Évreux. Après avoir adressé au conseil général de l'Eure, le 26 juillet 2010, une demande indemnitaire, laquelle demande a fait l'objet d'un rejet implicite, les époux B. vous demandent de condamner le conseil général de l'Eure à leur verser une somme de 50000 euros chacun, et une somme de 30000 euros à leur fille, $\mathrm{M}^{\text {lle }} \mathrm{S}$. B., en réparation du préjudice moral propre occasionné par les souffrances qui ont conduit au suicide d'E. B.

\section{Sur l'exception de prescription quadriennale}

Avant de vous prononcer sur la responsabilité du conseil général de l'Eure, vous devrez au préalable statuer sur l'exception de prescription quadriennale qu'il invoque. La partie défenderesse se fonde en effet sur les dispositions de l'article $1^{\mathrm{er}}$ de la loi du 31 décembre 1968 relative à la prescription des créances sur l'État, les départements, les communes et les établissements publics qui prévoient que « sont prescrites, au profit de l'État, des départements et des communes, sans préjudice des déchéances particulières édictées par la loi et sous réserve des dispositions de la présente loi, toutes créances qui n'ont pas été payées dans un délai de quatre ans à partir du premier jour de l'année suivant celle au cours de laquelle les droits ont été acquis [...]", et fait valoir que M. et $\mathrm{M}^{\mathrm{me}} \mathrm{B}$. ayant eu connaissance de leur créance le 15 février 2004, date à laquelle ils ont été informés des agressions subies par leur fille, leur action en réparation était prescrite au 31 décembre 2008, de sorte que leur demande indemnitaire présentée le 26 juillet 2010 est atteinte par la prescription quadriennale.

Toutefois, l'article 2 de la loi du 31 décembre 1968 prévoit que:

La prescription est interrompue par [...] Tout recours formé devant une juridiction relatif au fait générateur, à l'existence, au montant ou au paiement de la créance, quel que soit l'auteur du recours et même si la juridiction saisie est incompétente pour en connaître et si l'administration qui aura finalement la charge du règlement n'est pas partie à l'instance [...] Un nouveau délai de quatre ans court à compter du premier jour de l'année suivant celle au cours de laquelle a eu lieu l'interruption. Toutefois, si l'interruption résulte d'un recours juridictionnel, le nouveau délai court à partir du premier jour de l'année suivant celle au cours de laquelle la décision est passée en force de chose jugée. 
En vertu d'une jurisprudence constante, il résulte de ces dispositions qu'une plainte avec constitution de partie civile interrompt le cours de la prescription quadriennale dès lors qu'elle porte sur le fait générateur, l'existence, le montant ou le paiement d'une créance sur une collectivité publique (voyez notamment CE, 27 octobre 2006, département du Morbihan et autres, $\mathrm{n}^{\text {os }} 246931,247011,247071,247076$, fichés en A; CE, 11 avril 2008, M. Chatelain et autres, $\mathrm{n}^{0} 294767$, fiché en B).

Abandonnant sa jurisprudence Haudry du 10 octobre 2005, $\mathrm{n}^{\mathrm{O}} 264588$, fiché en $\mathrm{B}$, jugeant que les dispositions précitées de l'article 2 de la loi du 31 décembre 1968 subordonnent l'interruption du délai de prescription qu'elles prévoient en cas de recours juridictionnel à la mise en cause d'une collectivité publique et qu'une plainte contre $\mathrm{X}$, qui n'est pas expressément dirigée contre une collectivité publique, ne peut interrompre jusqu'à l'intervention d'une décision passée en force de chose jugée la prescription d 'une créance sur un établissement public hospitalier, le Conseil d'État a par la suite affirmé qu'il résulte de ces mêmes dispositions que, alors même que la juridiction judiciaire ainsi saisie n'est pas compétente pour connaître du fait générateur et même lorsque le responsable du dommage n'est pas partie à cette instance, un tel recours interrompt le cours de la prescription de la créance de la victime sur le responsable du dommage et jugé qu'une action engagée devant la juridiction pénale était de nature à interrompre la prescription de la créance sur un établissement public de santé même si cette action n'était pas dirigée expressément contre cet établissement (CE, 11 avril 2008, M. Chatelin et autres, $n^{0} 294767$ et CE, 26 mai 2010, Consorts Birien, $n^{0} 306617$, fichés en $\left.B\right)$.

Enfin, contrairement à ce qu'ont pu juger encore récemment, et pourtant à tort, certaines de vos cours (voyez notamment CAA Bordeaux, 6 septembre 2011, M. et $\mathrm{M}^{\text {me }}$ Gacougnolle, $\mathrm{n}^{0}$ 10BXoo828), le Conseil d'État a précisé que le fait que le recours devant une juridiction n'ait pas été introduit par le demandeur en réparation ne lui interdisait pas pour autant de s'en prévaloir, interprétation somme toute fort logique dès lors que l'article 2 de la loi du 31 décembre 1968 dispose que la prescription est interrompue par tout recours, quel qu'en soit son auteur (CE, 8 mars 2006, M. et $\mathrm{M}^{\mathrm{me}}$ Thuret, $\mathrm{n}^{\mathrm{o}}$ 270946, fiché en A, conclusions Rémi Keller).

En l'espèce, il résulte de l'instruction qu'à la suite de la prise de connaissance des faits dont leur fille E. avait été victime, $\mathrm{M}$. et $\mathrm{M}^{\mathrm{me}} \mathrm{B}$. ont déposé plainte avec constitution de partie civile le 31 mars 2004 auprès d'un juge d'instruction d'Évreux, pour les faits de viol concernant leur enfant. Ainsi, cette action, bien que n'étant pas expressément dirigée contre une personne publique, tendait à la recherche de l'établissement 
des responsabilités quant aux faits subis par leur fille E., et doit par conséquent être regardée comme relative au même fait générateur de la créance des requérants sur le conseil général de l'Eure, lequel avait la garde des mineurs concernés et employait les éducateurs chargés de leur surveillance, qui sont intervenus lors de la survenance de ces faits. Or, nous l'avons dit, $\mathrm{M}$. et $\mathrm{M}^{\mathrm{me}}$ B. ont eu connaissance de leur créance le 15 février 2004, date à laquelle, ainsi que l’admet le conseil général de l'Eure, ils ont été informés des agressions sexuelles subies par leur fille. Si la prescription a par conséquent commencé à courir le $1^{\mathrm{er}}$ janvier 2005 et expirait le 31 décembre 2008, les actions susmentionnées en constitution de partie civile ont été introduites le 31 mars 2004, avant l'expiration du délai de la prescription quadriennale, et ont eu pour effet, par conséquent, en vertu des dispositions de l'article 2 de la loi du 31 décembre 1968, d'interrompre ce délai. Ainsi, la CA de Rouen ayant rendu une ordonnance de non-lieu le 18 février 2010, ayant force de chose jugé, la créance de $\mathrm{M}$. et $\mathrm{M}^{\mathrm{me}} \mathrm{B}$. n'était pas prescrite le 26 juillet 2010, date de la demande d'indemnisation dont ils ont saisi le conseil général de l'Eure, la prescription ne commençant à courir qu'à partir du $1^{\text {er }}$ janvier 2011 en application des dispositions susmentionnées de l'article 2 de la loi du 31 décembre 1968. En outre, compte tenu de ce que nous avons dit précédemment, la circonstance que $\mathrm{M}^{\text {lle }} \mathrm{S}$. B. ne se soit pas constituée partie civile est sans incidence sur l'effet interruptif de la prescription à son égard, cette prescription étant interrompue par tout recours, quel que soit son auteur.

Dans ces conditions, nous vous proposons d'écarter l'exception de prescription quadriennale opposée par le conseil général de l'Eure.

\section{Sur la responsabilité du conseil général de l'Eure}

\subsection{En ce qui concerne le fait générateur de responsabilité}

Les époux B. et leur fille vous demandent à titre principal, de constater les fautes commises par le foyer départemental de l'enfance et le foyer du Bois-Verlande dans la prise en charge de $\mathrm{M}^{\text {lle }} \mathrm{E}$. B., et de reconnaître la responsabilité du conseil général de l'Eure dans les faits subis par celle-ci ou, à titre subsidiaire, de constater la responsabilité du conseil général de l'Eure sur le fondement de la responsabilité sans faute, en sa qualité de gardien, du fait des agissements commis par les mineurs ayant agressé $\mathrm{M}^{\text {lle }}$ E. B.

Vous pourriez être tentés de vous placer sur le terrain de la responsabilité sans faute invoquée à titre subsidiaire par les requérants 
pour condamner le conseil général de l'Eure. En effet, vous savez que transposant la jurisprudence de la cour de cassation, le Conseil d'État a consacré une nouvelle hypothèse de responsabilité sans faute fondée sur la notion de garde en considérant, dans son arrêt GIE Axa Courtage du 11 février 2005 ( $\mathrm{n}^{\mathrm{0}} 252169$, fiché en $\left.\mathrm{A}\right)$, que:

La décision par laquelle le juge des enfants confie la garde d'un mineur, dans le cadre d'une mesure d'assistance éducative prise en vertu des articles 375 et suivants du code civil, à l'une des personnes mentionnées à l'article 375-3 du même code, transfère à la personne qui en est chargée la responsabilité d'organiser, diriger et contrôler la vie du mineur; qu'en raison des pouvoirs dont l'État se trouve ainsi investi lorsque le mineur a été confié à un service ou établissement qui relève de son autorité, sa responsabilité est engagée, même sans faute, pour les dommages causés aux tiers par ce mineur.

Cette responsabilité a par la suite été étendue au département (CE, 13 février 2009, département de Meurthe-et-Moselle, $n^{0}$ 294265, fiché en B) puis aux mineurs délinquants faisant l'objet d'un placement en application de l'ordonnance du 2 février 1945 (CE, 13 novembre 2009, garde des Sceaux, ministre de la Justice, $\mathrm{n}^{0} 306517$, fiché en A).

Cependant, deux raisons nous conduisent à vous proposer de ne pas retenir ce fondement. Premièrement, nous ne sommes pas absolument certain que le Conseil d'État ait accepté de transposer le régime de la responsabilité sans faute fondée sur la notion de garde, consacré à propos des dommages causés aux tiers, à l'hypothèse où le préjudice est causé à des usagers ou, plus précisément, à des co-usagers du service, comme c'était le cas de $\mathrm{M}^{\text {lle }} \mathrm{E}$. B. dans le dossier qui vous est soumis. S'il est en effet certain que la responsabilité sans faute fondée sur le risque spécial ne peut être appliquée aux usagers du service mais uniquement aux tiers, l'arrêt rendu par le CE le 17 décembre 2010 (garde des Sceaux, ministre de la Justice et des Libertés, $\mathrm{n}^{\mathrm{O}} 334797$, fiché en A, conclusions M. Guyomar) laisse planer une incertitude sur cette question, quoique les conclusions rendues en l'espèce par M. Guyomar plaident plutôt en faveur de l'application de la responsabilité sans faute pour garde aux co-usagers du service. Mais, deuxièmement, il reste néanmoins que la responsabilité sans faute d'une personne publique ne doit être envisagée, vous le savez, qu'à titre subsidiaire, en l'absence de toute possibilité d'engagement de la responsabilité pour faute. Or il nous semble qu'en l'espèce, le conseil général de l'Eure a commis des agissements fautifs de nature à engager sa responsabilité.

En premier lieu, vous commencerez par rappeler à la partie défenderesse que l'autorité de la chose jugée en matière pénale ne s'attache qu'aux décisions des juridictions de jugement qui statuent sur le fond 
de l'action publique et non aux ordonnances de non-lieu que rendent les juges d'instruction, quelles que soient les constatations sur lesquelles elles sont fondées (voyez, par exemple, CE, 5 octobre 2011, $\mathrm{M}^{\mathrm{me}}$ Joseph, $\mathrm{n}^{\mathrm{o}} 337353$ ). Ainsi, l'ordonnance de non-lieu rendue le 29 octobre 2009 par le juge d'instruction, confirmée par la chambre d'instruction de la cour d'appel de Rouen le 18 février 2010, qui n'a pas l'autorité de la chose jugée et qui repose sur le fait que les éléments constitutifs des infractions de viol commis sur la personne d'un mineur de quinze ans en réunion, complicité de viol commis sur la personne d'un mineur de quinze ans en réunion, non-assistance à personne en danger et nondénonciation de crime ne sont pas suffisamment établis, ne fait pas obstacle, contrairement à ce que soutient le conseil général de l'Eure, à ce que soit poursuivie l'action en recherche de responsabilité devant la juridiction administrative, fondée non sur les poursuites pénales liées à l'affaire, mais sur les fautes reprochées au défendeur.

En second lieu, il résulte de l'instruction que la jeune E. B. a été confiée en urgence à l'aide sociale à l'enfance de l'Eure sur décision du procureur de la République, le 6 février 2004, dans le cadre de la procédure prévue à l'article 375-5 du code civil, eu égard aux fugues qui la mettaient en danger. Lorsqu'elle a intégré ce foyer au sortir d'une hospitalisation due à des tentatives de suicide, sa mère a attiré l'attention du foyer départemental de l'enfance sur ses intentions de fuguer et ses tendances à l'automutilation. Néanmoins, après avoir effectué une tentative de suicide et plusieurs fugues, E. B. a pu quitter le foyer départemental de l'enfance d'Évreux le 13 février 2004 en soirée et ne le rejoindre que le lendemain matin suivant, de son seul gré. En effet, aucun éducateur ou agent de ce foyer n'est intervenu au cours de cette soirée et de cette nuit afin de mettre fin à sa fugue. Certes, trois éducateurs membres du personnel éducatif du foyer du Bois-Verlande se sont rendus dans l'hôpital désaffecté afin d'en rapatrier les quatre garçons auteurs des actes à caractère sexuel, mais ils se sont bornés à prendre des mesures dépourvues d'effet, dès lors qu'au cours de cette même nuit, ces garçons ont de nouveau échappé à leur surveillance, et surtout les intéressés n’ont pris aucune mesure relative aux deux jeunes filles présentes sur les lieux, dont la jeune E. En outre, il n'est pas sérieusement contesté que les personnels du foyer du Bois-Verlande, auxquels étaient confiés des mineurs difficiles, étaient dépourvus de tout titre d'éducateur et ne disposaient d'aucune instruction de leur hiérarchie afin de gérer d'éventuelles, mais prévisibles, situations critiques. Il convient par ailleurs de souligner que l'hôpital désaffecté où se sont déroulés les faits litigieux était connu des mineurs confiés à l'aide sociale à l'enfance autant que du personnel 
éducatif comme un lieu de rencontres et de rapports sexuels entre filles et garçons des deux foyers concernés, lesdites pratiques étant peu ou prou tolérées par l'établissement, qui n'a en tout cas pris aucune mesure afin d'y mettre un terme. Ainsi, eu égard au profil très spécifique de la jeune E., dont avait connaissance le foyer départemental de l'enfance supposé assurer sa protection, et aux dysfonctionnements majeurs du service de l'aide sociale à l'enfance que nous venons de présenter, il nous semble que les requérants sont fondés à soutenir que le conseil général de l'Eure, chargé de cette mission, a vu sa responsabilité engagée en raison des fautes commises quant aux faits dont leur fille a été victime dans la nuit du 13 au 14 février 2004.

\subsection{Sur le préjudice et le lien de causalité}

Vous savez qu'une personne publique ne doit réparer le dommage résultant d'un événement qui lui est imputable que s'il existe un lien de causalité suffisamment direct entre cet événement et le préjudice subi par les demandeurs.

En l'espèce, si le préjudice moral propre subi par les requérants, résultant de la perte d'un être cher, nous parait indiscutable, le conseil général de l'Eure soutient que le lien de causalité entre les agressions sexuelles dont a été victime la jeune $\mathrm{E}$. et son suicide fait défaut dès lors que, lorsqu'elle a mis fin à ses jours le 17 mai 2005, elle se trouvait sous la garde de ses parents depuis plus d'une année. La partie défenderesse se prévaut, en outre, de la personnalité très fragile de la jeune fille, dont les tendances auto-mutilatrices et suicidaires se sont manifestées dès avant son placement au sein du foyer départemental de l'enfance et qui avait déjà fait l'objet d'autres agressions sexuelles, dont un viol.

Néanmoins, sans sous-estimer le poids de ces éléments, nous rappellerons que, comme le juge judiciaire, vous appréciez le lien de causalité non sur la base de la théorie dite de "l'équivalence des conditions", selon laquelle tous les événements ayant contribué au dommage sont pris en compte de manière identique, mais par référence à la théorie dite de la "causalité adéquate», méthode sélective en vertu de laquelle ne sont retenus que les faits ayant une vocation particulière à provoquer le dommage. Or en l'espèce, nous avons l'intime conviction que l'agression sexuelle multiple dont a été victime la jeune E., et qui n'a été rendue possible que par les dysfonctionnements du service public de l'aide sociale à l'enfance de l'Eure, a constitué, sinon la cause exclusive, du moins la cause principale de son suicide le 17 mai 2005, lequel est intervenu après un événement déclencheur, son audition un peu plus 
d'un mois avant, le 12 avril 2005, dans le cadre de l'instruction pénale ouverte dans cette affaire.

Nous vous proposons par conséquent de condamner le conseil général de l'Eure à réparer le préjudice moral subi par $M$. et $M^{\text {me }} B$., ainsi que leur fille $\mathrm{S}$., dont il sera fait une juste appréciation, en prenant en considération la personnalité de la jeune fille, dont les tendances automutilatrices et suicidaires se sont manifestées dès avant son placement au sein du foyer départemental de l'enfance, en le fixant à 15 ooo euros pour chacun d'eux. Enfin, nous vous proposons de mettre à la charge du conseil général de l'Eure une somme de 2 ooo euros au titre de l'article L 761-1 du Code de justice administrative.

Tel est le sens de nos conclusions.

Premier conseiller 